Correspondencia a:

Lenny Mariela Valdivia Peredo ${ }^{1}$

Rocio Ailen Ponce Nageda Alejandra Caero Maldona$\mathrm{da}^{1}$

Yeime Cervantes Bustamante $^{1}$

Mario Caceres.

${ }^{1}$ Estudiantes de Bioquimica y Farmacia de la Universidad Técnica Privada Cosmos.

Cochabamba - Bolivia

${ }^{2}$ Guia, Director de Investigación Nacional de la Universidad Técnica Privada Cosmos.

Cochabamba Bolivia

mcaceres_dii@unitepc. edu.bo

Procedencia y arbitraje: no comisionado, sometido a arbitraje externo

Recibido para publicación: 2 de junio de 2017 Aceptado para publicación: 04 de agosto de 2017

Citar como: Re Ci Sa UNI 2017;3(2):20-27

\section{PLANIFICACIÓN FAMILIAR Y EL ÉXITO DEL NUEVO SER}

\section{FAMILY PLANNING AND THE SUCCESS OF THE NEW BEING}

\section{PLANEJAMENTO FAMILIAR E O SUCESSO DO NOVO SER}

\section{RESUMEN.}

La utilidad de la planificación familiar, ayudará a las familias en la formación del nuevo ser, capacitándolos para ser más eficientes al tener un mayor apoyo entre padres. La principal tarea a partir de la lectura del artículo, es ayudar a reducir los embarazos no deseados, el abandono de los niños y a su vez los abortos. La planificación familiar es un componente esencial en el cuidado de la salud primaria y reproductiva. Si la práctica sexual se da sin planificación ni protección necesaria, se convierte en una amenaza que deriva en problemas como: deserción escolar, altas tasas de fecundidad por embarazos no planificados, o en otros casos terminan en aborto o muertes maternas e infantiles, como también enfermedades de transmisión sexual. La planificación familiar es considerada importante al facilitar a las parejas los métodos y medios con los cuales puedan tener hogares más estables y felices.

Palabras claves: planificación familiar, morbimortalidad y mortalidad materna, salud sexual y reproductiva.

\section{ABSTRACT.}

the formation of the new being, enabling them to be more efficient by having greater support among parents. The main task from reading the article, is to help reduce unwanted pregnancies, the abandonment of children and in turn abortions. Family planning is an essential component in the care of primary and reproductive health. If the sexual practice is given without planning or necessary protection, it becomes a threat that results in problems such as: school dropout, high fertility rates due to unplanned pregnancies, or in other cases they end in abortion or maternal and child deaths, as well as sexually transmitted diseases. Family planning is considered important in providing couples with the methods and means by which they can have more stable and happy homes.

Keywords: family planning, morbidity and mortality and mater- 
nal mortality, sexual and reproductive health.

RESUMO.

A utilidade do planejamento familiar ajudará as famílias na formação do novo ser, possibilitando que sejam mais eficientes por terem maior apoio entre os pais. A principal tarefa da leitura do artigo é ajudar a reduzir as gravidez indesejadas, o abandono de crianças, e por sua vez, abortos. O planejamento familiar é um componente essencial no cuidado da saúde primária e reprodutiva. Se a prática sexual é dado sem planejamento ou proteção necessária se torna uma ameaça, resultando em problemas, como: taxas de abandono escolar, altas taxas de fertilidade gravidez não planejada ou em outros casos que terminam em aborto ou mortes maternas e infantis, bem como doenças sexualmente transmissíveis. O planejamento familiar é considerado importante para fornecer aos casais os métodos e meios pelos quais eles podem ter llugares mais estáveis e felizes.

Palavras-chave: planejamento familiar, morbidade e mortalidade e mortalidade materna, saúde sexual e reprodutiva.

\section{INTRODUCCIÓN.}

La planificación familiar es considerada un componente esencial en el cuidado de la salud primaria y reproductiva, dado que juega un papel principal en la morbimortalidad materna y del recién nacido, como así también en la transmisión de enfermedades sexuales; del mismo modo, trae beneficios para la salud de las mujeres, de los niños, de la familia y de la comunidad en general. La utilización de anticonceptivos reduce la mortalidad materna y mejora la salud de las mujeres al prevenir los embarazos no deseados y los de alto riesgo; además de reducir la necesidad de abortos practicados sin garantías de inocuidad. ${ }^{1}$

La planificación familiar trae beneficios para la salud de la familia y de la comunidad, al facilitar a las parejas los medios para tener hogares menos numerosos y más sanos; por otro lado, reduce el incremento económico y emocional de la integración familiar. A pesar del aumento en la utilización de anticonceptivos en Cochabamba - Bolivia, existen necesidades de planificación familiar desatendidas entre las adolescentes, las mujeres adultas solteras sexualmente activas y las casadas (incluidas las que viven en unión libre). ${ }^{1}$ Es primordial que el Ministerio de Salud del Estado Plurinacional de Bolivia, proporcione el desarrollo de habilidades para la oferta de información adecuada en planificación familiar, a manera de atender y conocer las necesidades de las familias bajo la perspectiva de los derechos sexuales y reproductivos, así como la actualización continua de la información reproductiva. La información adecuada en planificación familiar es de fundamental importancia, ya que posibilita a las familias ejercer sus derechos, reconocer métodos anticonceptivos y hacer elecciones con autonomía, considerando abarcar orientaciones sobre métodos, así como salud sexual y reproductiva. Además de eso, los servicios de salud deben disponer de métodos y técnicas para el control de la fecundidad. ${ }^{2}$ La oferta de métodos anticonceptivos para 
las familias, con el objetivo de la elección autónoma, apunta a la necesidad que el Ministerio de Salud proporcionen una amplia gama de opciones, para que las familias puedan elegir libremente, de forma segura y confiable el método más adecuado, para los diferentes momentos de su vida reproductiva, de acuerdo con su historia de la salud y adaptación. ${ }^{3}$ La planificación familiar (PF) y la anticoncepción (AC) constituyen una base fundamental de la salud reproductiva (SR) dados sus potenciales beneficios sociales y de salud. Dichos beneficios incluyen desde la reducción de gastos económicos y la mortalidad materna e infantil ${ }^{1}$, hasta una mejora en la calidad de vida, lo que se traduce en mayores oportunidades de educación y empleo, además de la inserción más igualitaria de las mujeres a la sociedad. Asimismo, la PF, contribuye de manera importante al logro de varias Metas del Milenio. ${ }^{4}$

Lo más importante para una pareja que desea tener un hijo es lograr un embarazo satisfactorio y el nacimiento de un bebé sano en el seno de una familia feliz. El proceso de la reproducción humana tiene implicaciones para el individuo, las familias y los pueblos, están muy relacionados con el nivel socioeconómico y la cultura de los diferentes países. El mejor momento para tener un hijo supone una salud reproductiva que la Organización Mundial de la Salud (OMS) define como "el estado de completo bienestar físico, mental y social y no solamente la ausencia de enfermedad durante el proceso de reproducción". ${ }^{5}$

Aun cuando el período fértil en la mujer se extiende desde la menarquía hasta la menopausia, la edad óptima para la procreación se restringe a unos años de la vida, y varía según el criterio de diferentes autores: 20 a 29, 20 a 24 y 25 a 29 años(5). Se califica de riesgosa cuando la mujer tiene menos de 18 años, ya que tanto su madurez biológica y psicológica como su desarrollo económico y social son insuficientes para asumir la maternidad, con riesgo de la ocurrencia de bajo peso al nacer y de mortalidad para la madre y el niño. También cuando la mujer es mayor de 35 años, ya que en este período comienza el envejecimiento del sistema reproductor con el riesgo de trastornos uterinos, requerimientos de cesárea, o la aparición de malformaciones congénitas. Los productos radiactivos y algunos químicos afectan el desarrollo del feto, por lo que deben estar bien controlados y tenidos en cuenta en el caso de las personas en edad fértil que estén expuestas y deseen reproducirse. Además tienen riesgos las mujeres o parejas, cuya conducta personal y social constituya un peligro evidente para su salud y la de sus hijos, si conciben bajo esas circunstancias. ${ }^{5}$ En el presente artículo, se pretende concientizar a las familias sobre las estrategias existentes para la planificación familiar y el éxito del nuevo ser, mediante información que se deberá proporcionar a las familias, con la finalidad de brindar un mejor futuro al nuevo ser que se desea tener.

\section{METODOLOGÍA.}

Para la localización de los documentos bibliográficos se utilizaron varias fuentes documentales, puesto que su construcción corresponde a un artículo de revisión. Se realizó una búsqueda bibliográfica entre abril y mayo de 2017 a través de"google académi- 
co" utilizando los descriptores: planificación familiar, morbimortalidad materna, salud primaria y reproductiva, el nuevo ser humano del futuro. Los registros obtenidos oscilaron entre 32 y 26 tras la combinación de las diferentes palabras clave. También se realizó una búsqueda general en internet explorer con el buscador de "google" con los mismos términos. Se seleccionaron aquellos documentos que informasen sobre los aspectos formales que debían contener una revisión, la lectura crítica de documentos, las etapas de realización de una revisión bibliográfica. El objetivo de este estudio, es de dar a conocer a la sociedad la importancia de la planificación familiar, para un mejor futuro del nuevo ser, el cual rinda mejores expectativas y logros en la sociedad.

\section{RESULTADOS.}

El nuevo enfoque de la planificación familiar permite a la pareja definir el momento más apropiado para tener hijos, tomando en cuenta la salud de la mujer y los diferentes factores de riesgo reproductivo y los aspectos sociales que los rodea. ${ }^{12} \mathrm{En}$ el contexto del nuevo concepto de salud sexual y reproductiva, la planificación familiar se convierte en uno de los elementos más importantes en esta área, ya que permite a la pareja y a la mujer en particular, hacer uso de sus derechos sexuales y reproductivos para escoger libre y responsablemente el número y espaciamiento de sus hijos. ${ }^{13} \mathrm{La}$ planificación familiar bajo este enfoque favorece la libre decisión sobre el comportamiento sexual y reproductivo, incidiendo favorablemente sobre el bienestar y salud de las personas y de la familia. ${ }^{14}$

Es importante tomar en cuenta la utilización de dos tipos de métodos de planificación familiar: Es importante conocer toda la información necesaria para una adecuada planificación familiar esencial para lograr el bienestar de la pareja, la autonomía de las personas, apoyar a la salud y mejorar el desarrollo y evolución de la sociedad. ${ }^{14}$

Para ello es indispensable conocer los métodos anticonceptivos que existen en la planificación familiar, los métodos anticonceptivos naturales son aquellas técnicas que permiten a una pareja, mediante la observación de procesos relacionados con la ovulación, si desea evitar un embarazo sin utilizar ningún procedimiento artificial. ${ }^{15,16}$ Entre sus desventajas encontramos que no son muy confiables, en tanto se basan en patrones menstruales regulares sin tener en cuenta factores que puedan alterarlos. Además, ninguno ofrece protección contra el SIDA y otras enfermedades de transmisión sexual ${ }^{17}$.

En la planificación familiar natural se trata de averiguar la fecha más probable de ovulación con el fin de evitar el coito en esos días. Esto requiere que la pareja se abstenga de tener relaciones sexuales, por lo menos, durante 8 días en la mitad del ciclo menstrual, si se añaden los 4 a 6 días de menstruación resulta que el coito se debe evitar casi la mitad de cada mes. La práctica de este método es cada vez menor, porque, a pesar de ser el único método aceptado por la Iglesia Católica y de que cada nueva. ${ }^{7,18,19}$

En sí ataca el control artificial de la natalidad, las mujeres católicas cada vez más se 
inclinan hacia el uso de otros métodos más efectivos. La principal dificultad para la práctica de la planificación familiar natural, la constituye el hecho de que la ovulación ocurre 14 días antes del comienzo de la próxima regla, pero no necesariamente 14 días después del primer día de la menstruación. 7,20,21

El que utiliza estos métodos reconocen los cambios fisiológicos que ocurren durante el ciclo menstrual, porque están más conscientes de la responsabilidad de su maternidad. Los métodos de planificación familiar como ser los anticonceptivos contribuyen a posponer los matrimonios, una tendencia que ayuda tanto a las mujeres como a los hombres a encontrar parejas estables y atractivas desde el punto de vista económico; hay más posibilidades de que una relación se rompa tras un embarazo o parto no planificado que cuando estos se han planificado.22,23. La planificación familiar en Bolivia evitaría embarazos no deseados, ya que muchos niños son abandonados o asesinados (infanticidios) por sus padres o les impongan un padrastro o madrastra, los cuales en muchas ocasiones maltratan a los niños ocasionándoles daños físicos y psicológicos, que a la larga llega a ser un problema para que este niño se desenvuelva en su entorno. ${ }^{24,25}$

Si bien la desigualdad de género tiene muchas causas, hunde sus raíces en las normas socioculturales y mitos sobre lo que, en términos de comportamiento, está permitido a niños y hombres, pero no a niñas y mujeres, puesto que se incluyen aquí las creencias tradicional es relacionadas con la salud sexual y la planificación familiar. ${ }^{26,27}$

Por ejemplo, los estereotipos que relacionan la pasividad con las mujeres y la asertividad o agresividad con los hombres pueden atribuir a los hombres un mayor poder de decisión sobre cuestiones sexuales en una relación de pareja o dentro del matrimonio. De igual manera, otras normas vendrían a respaldar la idea de que la planificación familiar, incluidos los métodos anticonceptivos, es responsabilidad exclusiva de las mujeres y de las jóvenes. ${ }^{8}$ La participación informada de hombres, adolescentes y adultos, en programas de salud reproductiva y en la toma de decisiones, puede contribuir a socavar unas normas de género que son perniciosas, hasta el momento manteniéndose como un tabú que inclina su mirada hacia un solo género ${ }^{28}$. Estos programas también vendrían a reconocer que también los hombres y los niños tienen necesidades y responsabilidades en materia de salud reproductiva. La planificación familiar en Bolivia es necesaria para que ayude a las familias a lograr un mejor nivel de vida. ${ }^{29}$ A través de la planificación, las familias deciden cuantos hijos/as tener y cuándo tenerlos (evita los embarazos no deseados); posibilita a las mujeres que tengan sus hijos/as a las edades en que el embarazo ocasiona menores riesgos (entre 20 y 35 años), asegurando de esa manera la salud de la madre y la del bebé. Los servicios de planificación familiar son esenciales para la salud y la supervivencia de las comunidades de Bolivia, en particular mujeres y adolescentes. Cuando las mujeres están sanas, sus beneficios se repercuten en otras áreas del desarrollo humano, como por ejemplo la reducción de la pobreza, la educación, la población y la sostenibilidad de los recursos naturales. Se debe reforzar las políticas educativas para revertir embarazos no deseados en Bolivia. Hace falta trabajar en la difusión de los beneficios que gozan las mujeres al tener accesibilidad a obtener métodos anticonceptivos, a través 
de los medios masivos y además se debe incluir esta temática en la currícula escolar como una materia para que los estudiantes tengan una buena formación y con ello una óptima protección. ${ }^{9}$

\section{“EI SUMI otorga los métodos anticonceptivos a todas mujeres en edad fértil gra- tuitamente, pero lastimosamente muchas mujeres desconocen este derecho o no tienen educación en el uso de estos protectores". 9}

En la adolescencia se da un mayor índice de embarazos no planificados, en el que ocurren cambios en la esfera biológica, psicológica y social, que le confieren características especiales, los hace más vulnerables, puesto que tiene un impacto extraordinario en la esfera de la sexualidad. ${ }^{30}$ El problema de la falta de planificación familiar trae consigo consecuencias en diferentes ámbitos como los sociales, los de salud y económicos, Como consecuencia de ello, los niños pueden perder la vida debido a factores biológicos y no biológicos. Así pues, se produce un elevado índice de mortalidad por la misma falta de cuidado de los niños, la falta de alimentación adecuada a cada uno de ellos y el mal acceso al medio en el que se desarrollan aquellos.(10) Debido a que la conceptualización de salud definida por la Organización Mundial de la Salud (OMS), detalla:"es el estado de completo bienestar físico, mental y social, y no tan solo la ausencia de enfermedad o afección". ${ }^{31}$ El hombre es un ente social porque recibe influencias favorables y desfavorables como resultado de la interacción con la sociedad (constituida por semejantes a él) que lo rodea y con el medio ambiente físico, en el cual lleva a cabo su vida. ${ }^{11}$ La importancia de los aspectos emocionales que rodean el nacimiento de un hijo y que, muy a menudo, interfieren de manera negativa en la salud de la madre $y$, consecuentemente, en los cuidados del fututo hijo. ${ }^{32}$

\section{CONCLUSIONES.}

En la actualidad podemos reconocer que una Planificación Familiar, es una estrategia que tenemos a nuestro alcance, para disfrutar de una adecuada salud sexual y reproductiva como también de una herramienta fundamental para el desarrollo del nuevo ser y la integración a una sociedad cada vez más compleja. A pesar de la información que encontramos sobre los métodos anticonceptivos, es necesario implementar algún medio, que suministre de manera responsable la información adecuada de los diferentes y extensos métodos anticonceptivos que existen. Se impone intensificar la aplicación de políticas sanitarias dirigidas a perfeccionar la educación sexual y el acercamiento a los adolescentes, a fin de reducir las tasas de embarazos precoces, garantizar un mejor pronóstico de vida para la madre, el hijo y la familia, así como lograr una repercusión positiva en el desarrollo de la nueva sociedad. La planificación en Bolivia es muy importante debido a que en los últimos años se ve muchos niños sin hogar, esto es consecuencia de los embarazos no planificados, es muy urgente tratar esta necesidad con la ayuda del Ministerio de Salud de Bolivia, realizando campañas de concientización para motivar a las parejas a que puedan planificar un hogar estable y que el futuro de sus hijos sea más beneficioso tanto para la familia como para la sociedad. La aplicación de los métodos de la planificación familiar trae muchos beneficios para las familias y la sociedad, ya que, si un embarazo es planificado, la pareja 
estará consciente de la responsabilidad que tendrá que asumir con el hijo, les ayudará a prepararse psicológica y económicamente por anticipado. La finalidad de emplear este tipo de métodos es reducir el abandono de niños en la calle, los cuales por sus propios medios tienen que sobrevivir, muchas veces tomando malas decisiones o siendo mal influenciados, lo cual termina siendo un peligro para la sociedad.

\section{BIBLIOGRAFÍA.}

1. Liliana Franco Hincapié GMMZALMH. Planificación familiar en mujeres en condiciones de vulnerabilidad social. SALUD UIS. $2011 ;:$ p. 243

2. Pierre LAdS. Planificación familiar en Unidad de Salud de la Familia. Rev. Latino-Am. Enfermagem. 2010;: p. 2.

3. Luzia Aparecida dos Santos Pierre MJC. Planificación familiar en Unidad de Salud de la Familia. Rev. Latino-Am. Enfermagem. 2010;: p. 2.

4. Betania Allen-Leigh DeAAVHMeD. Inicio de vida sexual, uso de anticonceptivos. salud pública de méxico / vol. 55. 2013;: p. S236.

5. Alfonso CR. El mejor momento: reflexiones antes de tener hijos. Manual de diagnóstico y tratamiento en Obstetricia y Perinatología. 1997;: p. 1-2.

6. Durba IF. Metodos de Planificacion Familiar. Reproduccion Asistida. 2015.

7. Sam NAO. Planificación familiar, empoderamiento de la mujer e igualdad de genero. IPPF European Network. 2012.

8. Marciana-Soliz-Roca-. Más del $50 \%$ de bolivianas no sabe usar anticonceptivos. Salud sexual y reproductiva. 2013.

9. Garcia Oscarina. Planificacion Familiar. Guia multimedia informativa.

10. Risco DSD. Mortalidad Materna: Definicion. Cibersalud Ginecológica al día. 2010;: p. Comisión de Mujeres para los Refugiados. Paquete de servicios iniciales mínimos (PSIM) para la salud reproductiva en situaciones de crisis: Módulo de educación a distancia. Australia: 2011.

11. Eligibility Criteria for Contraceptive Use, 2010 Adapted from the World Health Organization Medical

12. Eligibility Criteria for Contraceptive Use, 4th edition. Editorial Board. Atlanta: 2010.

13. Instituto Nacional de Estadística e Informática. (2014). Encuesta Demográfica y de Salud Familiar

14. Ministerio de Salud. Documento Técnico: Promoviendo el Derecho a la Identificación para el Ejercicio del Derecho a la Salud, en el Marco del Aseguramiento Universal en Salud. Primera Edición. Lima: 2011.

15. Organización Mundial de la Salud. Planificación Familiar: Un Manual Mundial para Proveedores. Baltimore y Ginebra; 2011.

16. Organización Mundial de la Salud Medical Eligibility Criteria for Contraceptive Use. 4th Edition. 2015.

17. Department of Health and Human Services. Centers for Disease Control and Pre- 
vention. May 2010

18. U.S. Medical Eligibility Criteria for Contraceptive Use, Adapted from the World Health Organization

19. Medical Eligibility Criteria for Contraceptive Use, 4th edition. Mayo 2010.

20. Steiner M, López M, Grimes D, Cheng L, Shelton J, Trussel J, et al. Sino-implant (II) - a levonorgestrel-releasing two-rod implant: systematic review of the randomized controlled trials. Contraception 2010; 81(3): 197-201

21. Richardson A, Maltz F. Ulipristal acetate: eview of the efficacy and safety of a newly approved agent for emergency contraception. Clin Ther 2012; 34(1):24-36.

22. Brache V, Cochon L, Jesam C, Maldonado R, Salvatierra A, Levy D, et al. Immediate preovulatory administration of $30 \mathrm{mg}$ ulipristal acetate significantly delays follicular rupture. Hum Reprod 2010, 25(9): 2256-63

23. Cleland K, Raymond E, Westley E, Trussell J. Emergency Contraception Review: Evidence-based Recommendations for Clinicians. Clin Obstet Gynecol 2014; 57(4):743

24. Stratton P, Levens E, Hartog B, Piquion J, Wei Q, Merino M, et al. Endometrial effects of a single early luteal dose of the selective progesterone receptor modulator CDB2914. Fertil Steril 2010.

25. Bahamondes V, Hidago M, Bahamondes L,Monteiro I. Ease of insertion and clinical performance of the levonorgestrel-releasing intrauterine system in nulligravidas. Contraception 2011.

26. Ministerio de Salud Pública y Asistencia Social. Guía Técnica Para La Consejería en Planificación Familiar. 2016

27. Ministerio de Salud Pública y Asistencia Social. Guía de Atención en Planificación Familiar. 2014

28. Ministerio de Salud Pública y Asistencia Social. Norma Técnica de Planificación Familiar. 2014

29. Ministerio de Salud Pública y Asistencia Social. Normas de Atención Integral de Salud de Adolescentes. Octubre 2016

30. Ministerio de Salud Pública y Asistencia Social. Guía de Atención de los Principales Problemas de Salud de Adolescentes. Octubre 2016

31. Ministerio de Salud Pública y Asistencia Social. Protocolos de Atención para personas viviendo con VIH/SIDA. Noviembre 2015

32. Ministerio de Salud Pública y Asistencia Social. Guía de Atención a la Salud Sexual y Reproductiva del Adulto Masculino. Octubre 2015. 